\title{
Applications of a new $G$-invariant Implicit Function Theorem
}

\author{
LUTZ ReCKE \\ Humboldt University of Berlin, Unter den Linden 6, 10117 Berlin
}

\begin{abstract}
We formulate a result of the type of the Implicit Function Theorem for abstract equivariant equations, and we demonstrate by two examples (problems for ordinary and partial differential equations) how the assumptions can be verified and how the assertions can be interpreted.
\end{abstract}

Let $U$ and $V$ be Banach spaces, $\Lambda$ a normed vector space, $k \geq 2$ a natural number, $F: U \times \Lambda \rightarrow V$ a $C^{k}$-map and $u_{0} \in U$ a point such that $F\left(u_{0}, 0\right)=0$ and that

$$
L:=\partial_{u} F\left(u_{0}, 0\right) \text { is a Fredholm operator (index zero) from } U \text { into } V \text {. }
$$

Further, let $G$ be a compact Lie group which works linearly on $U$ and $V$, respectively, such that the maps $u \in U \mapsto \gamma \cdot u \in U$ and $v \in V \mapsto \gamma \cdot v \in V$ are continuous for all $\gamma \in G$, that the map $\gamma \in G \mapsto(\gamma \cdot u, \gamma \cdot v) \in U \times V$ is continuous for all $(u, v) \in U \times V$ and that

$$
F(\gamma \cdot u, \lambda)=\gamma \cdot F(u, \lambda) \text { for all } u \in U, \lambda \in \Lambda \text { and } \gamma \in G .
$$

Then the group orbit $\mathcal{O}\left(u_{0}\right):=\left\{\gamma \cdot u_{0}: \gamma \in G\right\}$ is a $C^{k}$-submanifold in $U$ (cf. [2]), and the tangential space $T_{u_{0}} \mathcal{O}\left(u_{0}\right)$ is a subspace of ker $L$. We assume that ker $L$ is as small as possible under these assumptions, i.e. that

$$
\operatorname{ker} L=T_{u_{0}} \mathcal{O}\left(u_{0}\right) \text {. }
$$

Let $G_{0}:=\left\{\gamma \in \Gamma: \gamma \cdot u_{0}=u_{0}\right\}$ be the isotropy subgroup of $u_{0}$ and $U_{0}:=\{u \in U$ : $\gamma \cdot u=u$ for all $\left.\gamma \in G_{0}\right\}$ and $V_{0}:=\left\{v \in V: \gamma \cdot v=v\right.$ for all $\left.\gamma \in G_{0}\right\}$ the corresponding fixed point subspaces. Then $L U_{0}$ is a closed subspace of finite codimension in $V_{0}$, and we denote its codimension in $V_{0}$ by $\operatorname{codim}_{V_{0}} L U_{0}$.

The following theorem was proved in [3]. It describes the solution behavior near $\mathcal{O}\left(u_{0}\right) \times\{0\}$ of equation

$$
F(u, \lambda)=0
$$

Theorem Let $\Lambda_{2}$ be a subspace in $\Lambda$ such that

$$
\operatorname{dim} \Lambda_{2}=\operatorname{codim}_{V_{0}} L U_{0} \text { and } V_{0}=L U_{0} \oplus \partial_{\lambda} F\left(u_{0}, 0\right) \Lambda_{2} .
$$

Further, let $\Lambda_{1}$ be a closed complement of $\Lambda_{2}$ in $\Lambda$.

Then there exist neighbourhoods $\mathcal{W} \subseteq U$ of $\mathcal{O}\left(u_{0}\right)$ and $\mathcal{W}_{j} \subseteq \Lambda_{j}$ of the zero's $(j=1,2)$ and $C^{k}-$ maps $\hat{u}: \mathcal{W}_{1} \rightarrow U_{0}$ and $\hat{\lambda}_{2}: \mathcal{W}_{1} \rightarrow \Lambda_{2}$ with $\hat{u}(0)=u_{0}$ and $\hat{\lambda}_{2}(0)=0$ such that for $u \in \mathcal{W}$ and $\left(\lambda_{1}, \lambda_{2}\right) \in \mathcal{W}_{1} \times \mathcal{W}_{2}$ it holds $F\left(u, \lambda_{1}+\lambda_{2}\right)=0$ if and only if $\lambda_{2}=\hat{\lambda}_{2}\left(\lambda_{1}\right)$ and $u=\gamma \cdot \hat{u}\left(\lambda_{1}\right)$ for some $\gamma \in \Gamma$. 
The so-called $G$-invariant Implicit Function Theorem of E. DANCER [2] is a special case of the theorem above, namely that of $\operatorname{codim}_{V_{0}} L U_{0}=0$. In that case the theorem states that for all small $\lambda$ there exists exactly one orbit of solutions to (2) near $\mathcal{O}\left(u_{0}\right)$. But if $\operatorname{codim}_{V_{0}} L U_{0}>0\left(\operatorname{codim}_{V_{0}} L U_{0}\right.$ can be any nonnegative integer up to $\left.\operatorname{dim} G-\operatorname{dim} G_{0}\right)$, then (2) is solvable near $\mathcal{O}\left(u_{0}\right) \times\{0\}$ if and only if $\lambda_{2}=\hat{\lambda}_{2}\left(\lambda_{1}\right)$, i.e if and only if $\lambda$ belongs to a $C^{k}$-submanifold in $\Lambda$ of codimension $\operatorname{codim}_{V_{0}} L U_{0}$. In other words: In order to have solutions $u \approx \mathcal{O}\left(u_{0}\right)$ to $(2)$, one can choose $\lambda_{1} \approx 0$ arbitrarily, but then $\lambda_{2}$ is determined by $\lambda_{1}$. In this spirit $\lambda_{1}$ is a "control" parameter and $\lambda_{2}$ a "state" parameter. In applications the role of condition (3) is to show how to split $\Lambda$ into subspaces of "control" and "state" parameters. Of course, such a splitting is not unique, in general.

Let us consider two examples. The results produced in the setting of these examples, are already known, of course. So the aim is to show how the assumptions (1) and (3) can be verified in simple, but typical for more complicated applications, examples, which type of assertions follows and that quite different "dynamic" and "static" problems fit in the abstract setting above.

Periodic traveling waves The problem of periodic traveling wave solutions to reaction-diffusion systems $\partial_{t} w=A \partial_{x}^{2} w+f(w), x \in \mathbb{R}, w \in \mathbb{R}^{n}$, leads, via the ansatz $w(t, x)=u(d x-c t)$, to the problem of $2 \pi$-periodic solutions to the system of ordinary differential equations $d^{2} A u^{\prime \prime}+c u^{\prime}+f(u)=0$. Here $f: \mathbb{R}^{n} \rightarrow \mathbb{R}^{n}$ is supposed to be smooth, and $A$ is a positive definite $n \times n$-matrix. Thus, we have the setting above with $U:=C_{2 \pi}^{2}\left(\mathbb{R}^{n}\right), V:=C_{2 \pi}\left(\mathbb{R}^{n}\right), \Lambda:=\mathbb{R}^{2}, F(u, c, d):=d^{2} A u^{\prime \prime}+c u^{\prime}+f(u)$, $G:=\mathbf{S O}(2) \approx \mathbb{R} / 2 \pi$ and $(\gamma \cdot u)(t):=u(t+\gamma)$. Suppose that $F\left(u_{0}, c_{0}, d_{0}\right)=0$, that $d_{0}>0$ and that $2 \pi$ is the minimal period of $u_{0}$. Then $U_{0}=U$ and $V_{0}=V$, and (1) is satisfied with $T_{u_{0}} \mathcal{O}\left(u_{0}\right)=\operatorname{span}\left\{u_{0}^{\prime}\right\}$ iff the eigenvalue zero of $\partial_{u} F\left(u_{0}, c_{0}, d_{0}\right)$ is geometrically simple. Further, we have $\partial_{c} F\left(u_{0}, c_{0}, d_{0}\right)=u_{0}^{\prime} \notin \operatorname{im} \partial_{u} F\left(u_{0}, c_{0}, d_{0}\right)$, and hence (3) with $\Lambda_{2}=\{(c, d): d=0\}$, iff the eigenvalue zero of $\partial_{u} F\left(u_{0}, c_{0}, d_{0}\right)$ is algebraically simple. Therefore, in this case the wave speed $c$ is locally determined by the spatial period $2 \pi / d$.

On the other hand, we have $\partial_{d} F\left(u_{0}, c_{0}, d_{0}\right)=2 d_{0} A u_{0}^{\prime \prime} \notin \operatorname{im} \partial_{u} F\left(u_{0}, c_{0}, d_{0}\right)$ iff it holds $\int_{0}^{2 \pi}\left\langle A u_{0}^{\prime \prime}, v\right\rangle d t \neq 0$ for all nonzero $2 \pi$-periodic solutions to the adjoint linearized equation $d_{0}^{2} A v^{\prime \prime}-c_{0} v^{\prime}+f^{\prime}\left(u_{0}\right)^{T} v=0$. Hence, in that case the spatial period is locally determined by the wave speed.

Now, let us consider solutions with $c=0$, i.e. periodic "frozen" or "standing" waves. In this case we have $\Lambda:=\mathbb{R}$ and $G:=\mathbf{O}(2)$, because $F(\cdot, 0, d)$ is $\mathbf{O}(2)$-equivariant (with the reflection $(\delta \cdot u)(t):=u(-t))$. Suppose again that $2 \pi$ is the minimal period of $u_{0}$, but now, additionally, assume that there exists a $\gamma \in \mathbf{S O}(2)$ such that $\delta \gamma \cdot u=\gamma \cdot u$. Then $V_{0}=\{v \in V: \delta \gamma \cdot v=\gamma \cdot v\}$ and $U_{0}=U \cap V_{0}$. If the eigenvalue zero of $\partial_{u} F\left(u_{0}, 0, d_{0}\right)$ is simple, then $\partial_{u} F\left(u_{0}, 0, d_{0}\right)$ is a Fredholm operator from $U_{0}$ into $V_{0}$ which is injective (because $\left.u_{0}^{\prime} \notin U_{0}\right)$. Hence, (3) is satisfied with $\Lambda_{2}=\Lambda$, and for all $d \approx d_{0}$ there exists exactly one orbit of symmetric periodic standing wave solutions near $\mathcal{O}\left(u_{0}\right)$.

Symmetric elliptic boundary value problems Consider the elliptic boundary value problem

$$
A \Delta u+f(u, \mu)=0 \text { in } \Omega, u=0 \text { on } \partial \Omega, u \in \mathbb{R}^{n}
$$


in a symmetric bounded domain $\Omega \subset \mathbb{R}^{2}$. Here $f: \mathbb{R}^{n} \times \mathbb{R} \rightarrow \mathbb{R}^{n}$ is supposed to be smooth, again, and $A$ belongs to the space $\mathbb{M}_{n}$ of $n \times n$-matrices. We have the setting above with $U:=W^{2,2}(\Omega) \cap W_{0}^{1,2}(\Omega), V:=L^{2}(\Omega), \Lambda:=\mathbb{M}_{2} \times \mathbb{R}, F(u, A, \mu):=$ $A \Delta u+f(u, \mu), G:=\mathbf{O}(2),(\gamma \cdot u)(r, \varphi):=u(r, \varphi+\gamma)$ for all $\gamma \in \mathbf{S O}(2)$ and $(\delta \cdot u)(r, \varphi):=$ $u(r,-\varphi)$. Suppose $F\left(u_{0}, A_{0}, 0\right)=0$ with a positive definite $A_{0}$, and let the eigenvalue zero of $\partial_{u} F\left(u_{0}, A_{0}, 0\right)$ be simple. Then (1) is satisfied with $T_{u_{0}} \mathcal{O}\left(u_{0}\right)=$ span $\left\{\partial_{\varphi} u_{0}\right\}$. Further, let $\frac{2 \pi}{n}$ be the minimal period of $u_{0}(r, \cdot)$ (with $n \in \mathbb{N}$ ). Then $G_{0}=\mathbf{D}_{n}$ (the dihedral group) or $G_{0}=\mathbf{Z}_{n}$ (the cyclic group) if there exists a $\gamma \in \mathbf{S O}(2)$ such that $\delta \gamma \cdot u_{0}=\gamma \cdot u_{0}$ or if not. In the first case we have, as in the example above, $\partial_{u} F\left(u_{0}, A_{0}, 0\right) U_{0}=V_{0}$ (because $\left.\partial_{\varphi} u_{0} \notin U_{0}\right)$. Hence, in that case (3) is satisfied with $\Lambda_{2}=\Lambda$, and for all $A \approx A_{0}$ and $\mu \approx 0$ there exists exactly one orbit of symmetric solutions near the orbit of $u_{0}$. In the second case we have $\partial_{\mu} F\left(u_{0}, A_{0}, 0\right) \in V_{0} \backslash \partial_{u} F\left(u_{0}, A_{0}, 0\right) U_{0}$ iff $\int_{\Omega}\left\langle\partial_{\mu} f\left(u_{0}, 0\right), v\right\rangle d x \neq 0$ for all nonzero solutions to the adjoint linearized boundary value problem $A \Delta v+\partial_{u} f\left(u_{0}, 0\right)^{T} v=0$ in $\Omega$, $v=0$ on $\partial \Omega$. Hence, in that case (3) is satisfied with $\Lambda_{2}=\{(A, \mu): A=0\}$, and only for all $A \approx A_{0}$, which satisfy an equation $A=\hat{A}(\mu)$, there exists an orbit of solutions near $\mathcal{O}\left(u_{0}\right)$.

In other words: If a solution to (4) is not radially symmetric, but has a reflection symmetry, then generically its orbit survives under all small perturbations of $A$ and $\mu$ as an orbit of solutions. In [1] a problem from elastostatics is analyzed in a similar setting, where all the radially nonsymmetric solutions (up to eight for appropriate parameters) have a reflection symmetry.

If a solution to (4) doesn't have a reflection symmetry, then generically it survives as an orbit of solutions only under quite special perturbations. If in that case (4) is the stationary problem of an evolution problem, for example of $\partial_{t} u=A \Delta u+f(u, \mu)=0$, it is natural to ask whether or not the orbit of $u_{0}$ survives under all small perturbations as an invariant manifold. But that's another question, of course.

Rotating and modulated waves In [3] it is shown how the abstract theorem above can be applied in order to describe the parameter dependence of rotating and of modulated wave solutions (especially of the wave frequencies and the modulation frequencies) to equivariant ordinary differential equations.

\section{References}

[1] E. Buzano and A. Russo, Bifurcation problems with $\mathbf{O}(2) \times \mathbb{Z}_{2}$ symmetry and the post-buckling behavior of a cylindrical shell, Annali Mat. Pura Appl. 146(IV) (1987), 217-262.

[2] E. N. DANCER, The G-invariant implicit function theorem in infinite dimensions II, Proc. Roy. Soc. Edinburgh A102 (1986), 211-220.

[3] L. Recke and D. Peterhof, Abstract forced symmetry breaking and forced frequency locking of modulated waves, J. Differ. Equat. 144 (1998), 233-262. 